\title{
Development of a web based GIS for health facilities mapping, monitoring and reporting: \\ A case study of the Zambian Ministry of health
}

Hilary Takudzwa Mushonga ${ }^{1}$, Faustin Banda ${ }^{2}$ and Augustine Mulolwa ${ }^{2}$

${ }^{1}$ Department of Mining Engineering, University of Zimbabwe, mushongaht@eng.uz.ac.zw

${ }^{2}$ Department of Geomatic Engineering, University of Zambia

\section{DOI: http://dx.doi.org/10.4314/sajg.v6i3.4}

\begin{abstract}
Around the world health professionals and authorities, in many cases, do not have the ability to visualize health related spatial information to make timely decisions. The high cost of deploying a desktop Geographical Information System (GIS) for Public Health management coupled with the need for specialised training in order to use geospatial tools have contributed to the low uptake of GIS as a decision support tool in public health management. Lack of a real time data collection and visualization tool for health facilities has in many cases led to late responses in situations where time critical decision had to be made. This research reviewed recent literature on GIS in health care with particular emphasis on web GIS technologies and how they can aid in analysing health care needs, access, and utilization to support in the planning and evaluation of new service locations as well as use of GIS in disease surveillance. This research is aimed at producing a web based GIS that can be used to collect data from health facilities and in turn provide this data to public health administrators to support decision making, it also focuses on creating a portal for public interaction with health facilities spatial information.
\end{abstract}

Key Words: Spatial Decision Support System, Web GIS, Mapping, Health geography

\section{Introduction}

Geographic Information Systems (GIS) is a geospatial technology and science that can be utilized to identify and display geographic patterns of disease, assess environmental exposures, and estimate incidence, prevalence, and survival statistics as well as expose health disparities to communicate clearly with the public, business and political leaders (Gotz et al., 2009). Public health management requires information on various aspects like the number of health facilities that are available and prevalence of diseases in order to take decisions on either creating additional infrastructure facilities or for taking immediate action to respond to situations. GIS is fast becoming a vital tool in healthcare applications covering database management, planning, risk assessment, service area mapping and location identification (Tahir, 2010). 
While there are several Decision Support Systems (DSS) in use by public health officials, many do not utilise geospatial technologies (Budge et al., 2006). Currently, the Zambian Ministry of Health has an initiative to introduce GIS into its operations, targeting the use of GIS in tasks such as location selection for development of new health facilities as well as tracking the spread of diseases. According to Church (2002) GIS can support a wide range of spatial queries that can be used to support location studies. Thus GIS has become an indispensable spatial decision support system (SDSS). The primary objective of this research was to develop a prototype health facilities web GIS that would facilitate data collection, visualisation and reporting. This system will also enable members of the public to access information on the location of health facilities and the services they offer.

\section{Background}

\subsection{Health Geography}

Health geography is an area of medical research that incorporates geographic techniques into the study of human health and the spread of diseases. The most notable early application of maps in health care is the use of a hand-drawn map by Dr John Snow in London in the mid 1850's to analyse the geographic location of deaths caused by cholera (Green, 2012). Upon plotting the geographic location of the deaths, Dr Snow realised most of them were clustered around the Broad street water pump. Once the pump handle was removed the cholera outbreak receded, though some scholars would argue that the epidemic was already on the decline. Health geography can also be traced to earlier times such as the time of the Greek doctor, Hippocrates (5th-4th centuries), where he studied the effect of location on human health (Elsabawy, 2013)

\subsection{Web based GIS}

GIS is a system of hardware, software, procedures and people that capture, store, edit, manipulate, manage, analyse, share, and display georeferenced data (Fu and Sun, 2011). GIS have been around from before the era of the internet, and according to Fu and Sun (2011) the first functional GIS was developed in 1962 by Roger Tomlinson for Canada's Federal Department of Forestry and Rural Development. The system was called the Canada Geographic Information System (CGIS). The purpose of a GIS is to provide a spatial framework to support decisions for the intelligent use of earth's resources and to manage the man-made environment (Zeiler, 1999). A web Based GIS Should be able to undertake any task that a conventional desktop GIS is able to do, but should be deployed on the World Wide Web. The Xerox PARC Map Viewer, developed by the Xerox Corporation Palo Alto Research Centre (PARC), is recognised as the first web GIS to be developed. The Xerox PARC Map Viewer was an experiment allowing retrieval of interactive information on the Web, rather than providing access to static files (Putz, 1994). A multitude of web based GIS have been developed for many applications including vehicle routing, crime mapping and utility 
mapping. Several developments have taken place in the road to modern day web based GIS systems, the first major development being the emergence of interactive web technologies in the web 2.0 era, beginning at the turn of the 21st century. Another huge development was the introduction of geo browsers / virtual globes the first of which was Google earth in 2005.

\section{GIS in Public Health Management}

There is great need for Health administrators, professionals and researchers to be trained and get user support in GIS technology, data and epidemiological methods in order to use GIS properly and effectively (Johnson and Johnson, 2001). The use of GIS in public health still remains primarily in the hands of technical specialists who themselves have no knowledge of public health matters, thus there is need to empower health administrators by providing GIS platforms that are simpler to operate. According to Glass et al., (1993) though simplified systems can be made to work on computers that are available to public health workers, these systems have limited flexibility and require the services of highly-skilled people. There are several application areas in public health management where GIS as a Spatial Decision Support System (SDSS) can be applied. According to Mesgari and Masoomi (2008) some of areas where GIS can be applied include the following:

- Management of Environmental Pollution.

- Disease Monitoring and Control

- Epidemiology and Health Related Disaster Management

\section{Materials and Methodology}

The online health facilities web based GIS system was designed and implemented using MySQL as the database management System. An Apache server was used as the application server. PHP was used as the scripting language to program the server side that manipulates the knowledge in the database. To ensure the successful implementation of the health facilities web based GIS, a development cycle based on the waterfall approach to system development was used. The waterfall approach makes it is easier to manage the system development because each phase of development has specific deliverables and thus can be evaluated separately. Figure 1 shows the system development cycle starting with the feasibility study up until the system maintenance stage. 


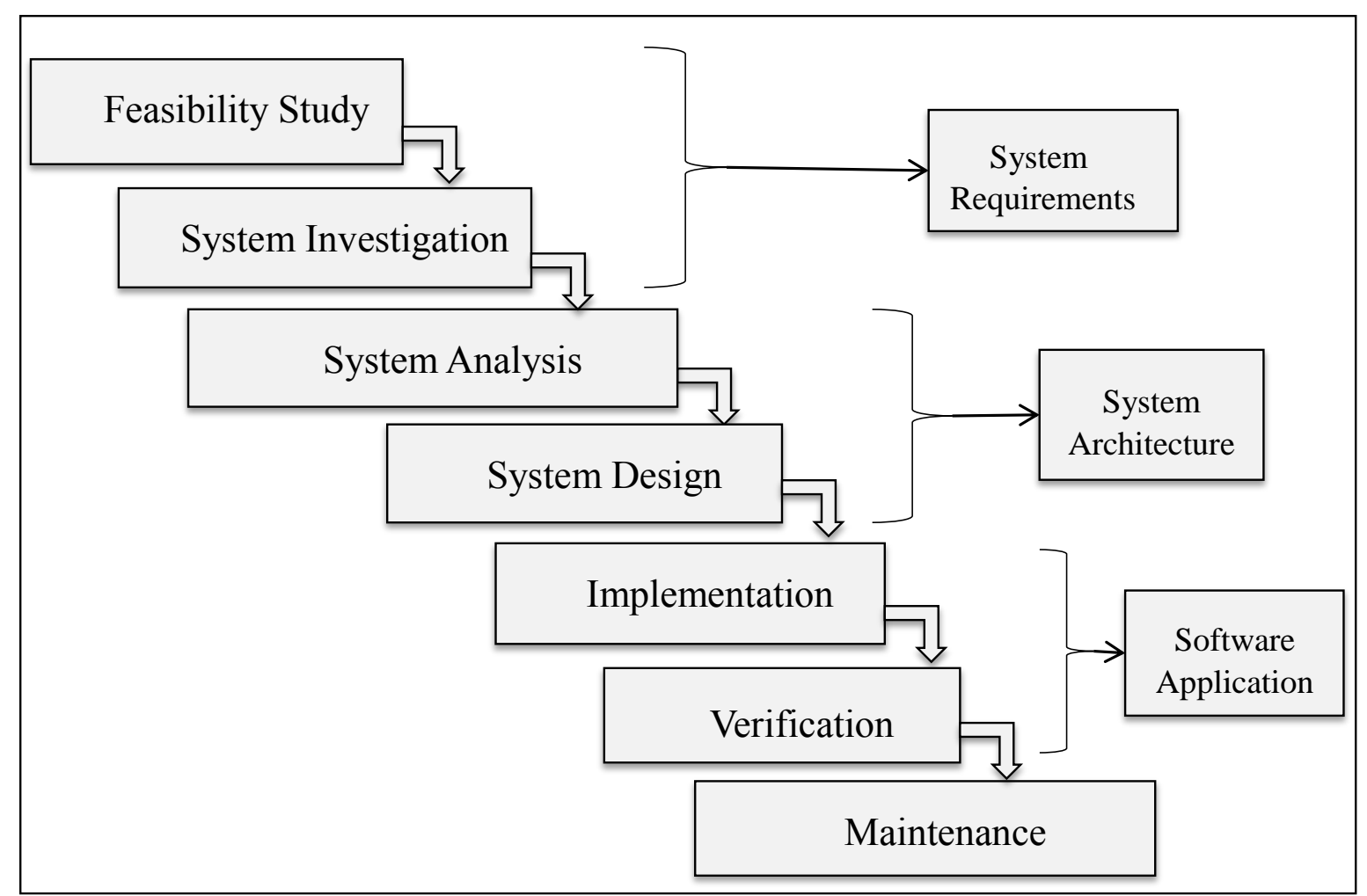

Figure 1. System Development Cycle

In determining the system requirements, interviews were conducted with officials from the Ministry of Health's Monitoring and Evaluation Department as well as the Disease Surveillance Department. The specifications determined from the requirements gathering phase were studied and used to guide the system design phase where the system architecture was developed. The system architecture then in turn mapped the way for development of the software application.

\section{Results}

\subsection{System Implementation}

The research produced an online health facilities web GIS application with a hybrid architecture where, spatial functions are conducted on the client side to minimise data traffic between the web server and browser thus ensuring smooth operation without processing lag. The system architecture is as shown in Figure 2. 


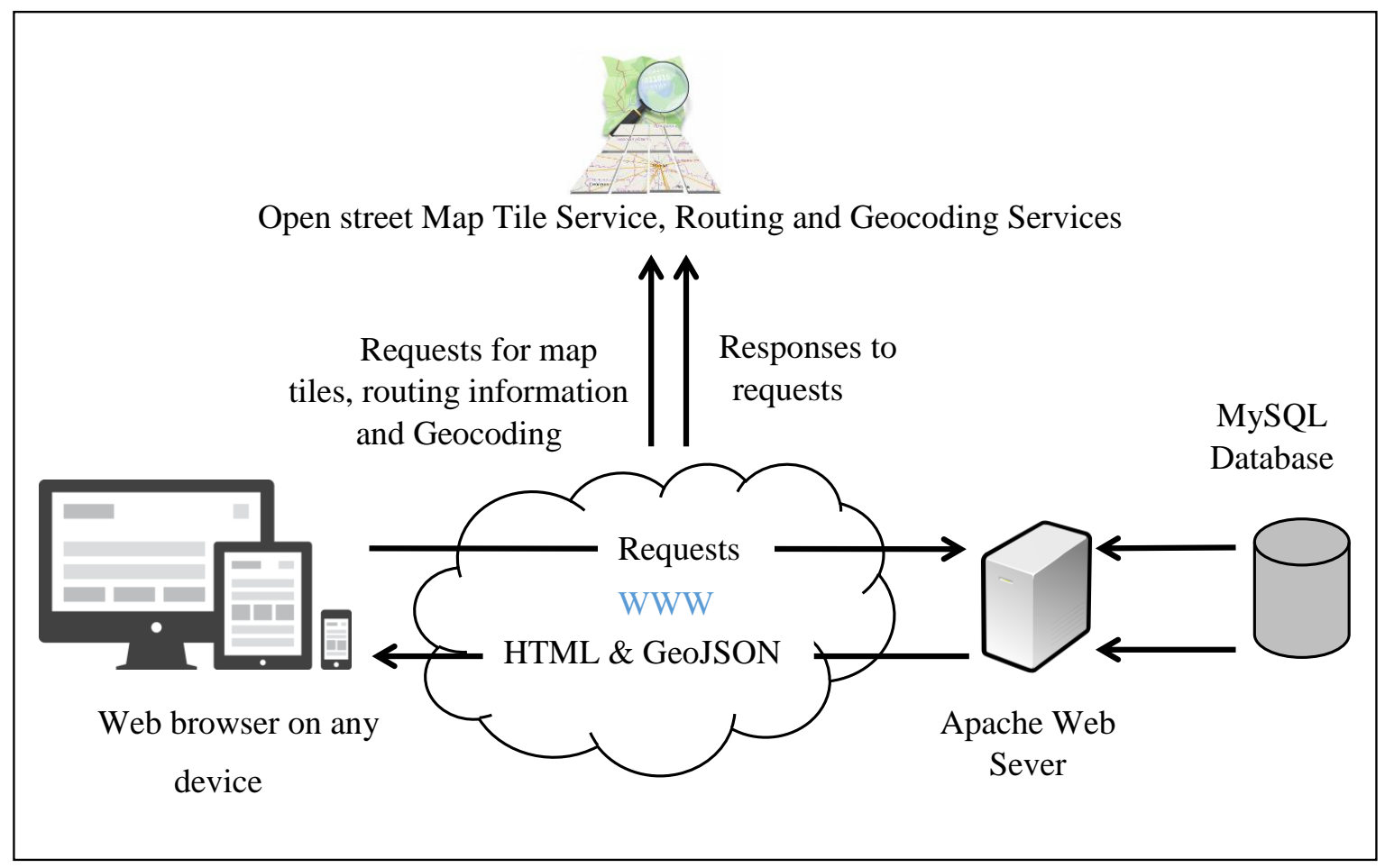

Figure 2. Health facilities web GIS architecture

On the server side of the system there is an apache server, which serves the application files to browsers and queries the database in response to requests made within the web GIS application. PHP, which is a server side scripting language, was used to produce scripts that query the MySQL database. PHP scripts query locations and convert results into GeoJSON (Geographic Java Script Object Notation) format, for display within the web application. GeoJSON, which is a lightweight data-interchange format, is less verbose than XML and ultimately results in lower data file size than alternatives such as Geography Markup Language (GML) or Keyhole Markup Language (KML) both of which are XML based. GeoJSON's lower file sizes which result in faster system load time and its simplicity that enables easy parsing of the geometry and fields led to its selection for use in the health facilities web based GIS. The web based GIS makes requests for map tiles from the open street map tile service; the map tiles form the base map for the system showing regional boundaries, roads and other points of interest. Other web map tile services such as Google maps and Bing maps can be integrated into the system to provide base maps but they have restrictions in the number of calls for tiles that can be made and require a license keys for use. Open street map tile service was selected for use because it has no restrictions and is free with an open license.

\subsection{User Interface design}

The research endeavored to produce a web GIS with a simple and intuitive interface that would enable first time GIS users to use the system with minimal instruction. The main interface together with descriptions of its main components is as shown in Figure 3. 


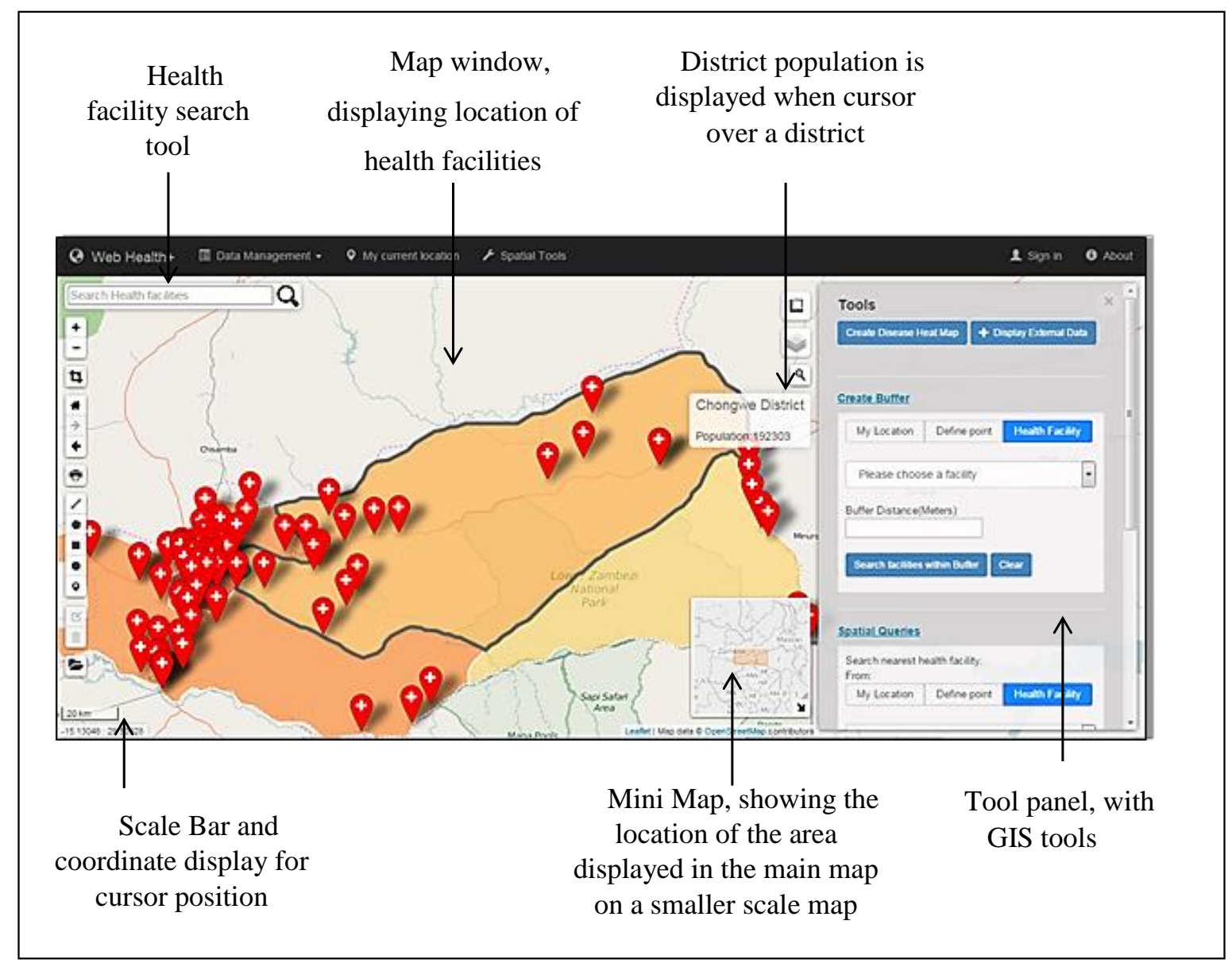

Figure 3. Main Window of the health facilities web GIS

The interface also includes a layer control that enables users to toggle layers from the display, provide zoom in and out as well navigation buttons. The system was built in such way that it is responsive and thus the interface maintains its display integrity and appears the same across screen sizes. Figure 4 shows how the system renders on a mobile device. 


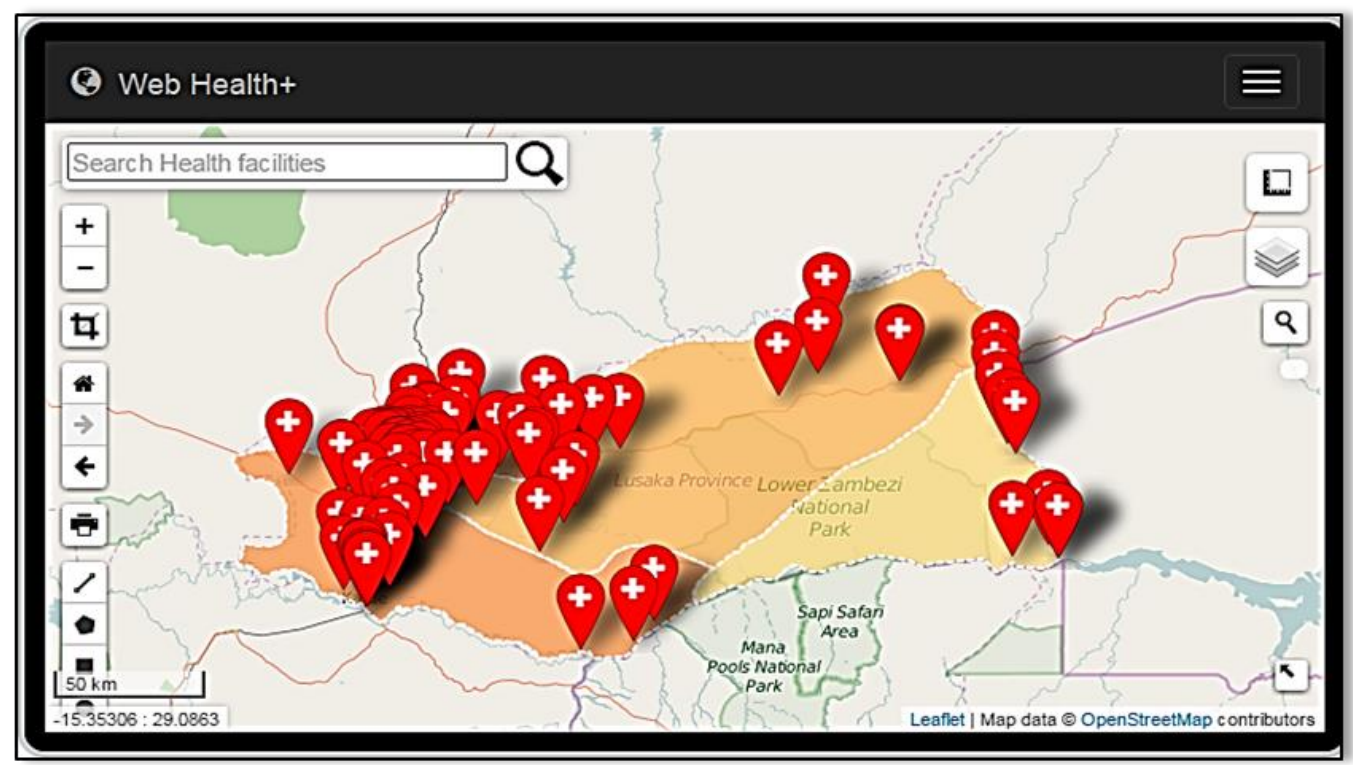

Figure 4. System Interface on a Mobile Device

\subsection{GIS Functionality within the web based GIS}

\subsubsection{Nearest Neighbour Search}

This tool enables the user to search for the nearest health facility from any one of the following:

i. The user's current location: The system uses IP (Internet protocol) geo-location on a desktop computer and GPS on mobile devices. Permission for the system to access location has to be granted within the web browser settings.

ii. A user defined point: The user can define a point by clicking on any location on the map.

iii. Another health facility: The user selects a health facility from a list of all available health facilities; the search is then run from the selected health facility.

This facility will aid in health facility location planning where planners can determine the closest facility and ultimately the distance to it.

\subsubsection{Routing}

The system has an integrated routing facility that leverages the open street map routing engine. The function enables the user to get a route between any health facility and the user's current location, a user defined point or another health facility. The system also provides turn by turn directions to guide users to their desired destination. This function will mainly aid both public health managers and members of the public in getting to health facilities. The routing function is shown below in Figure 5. 


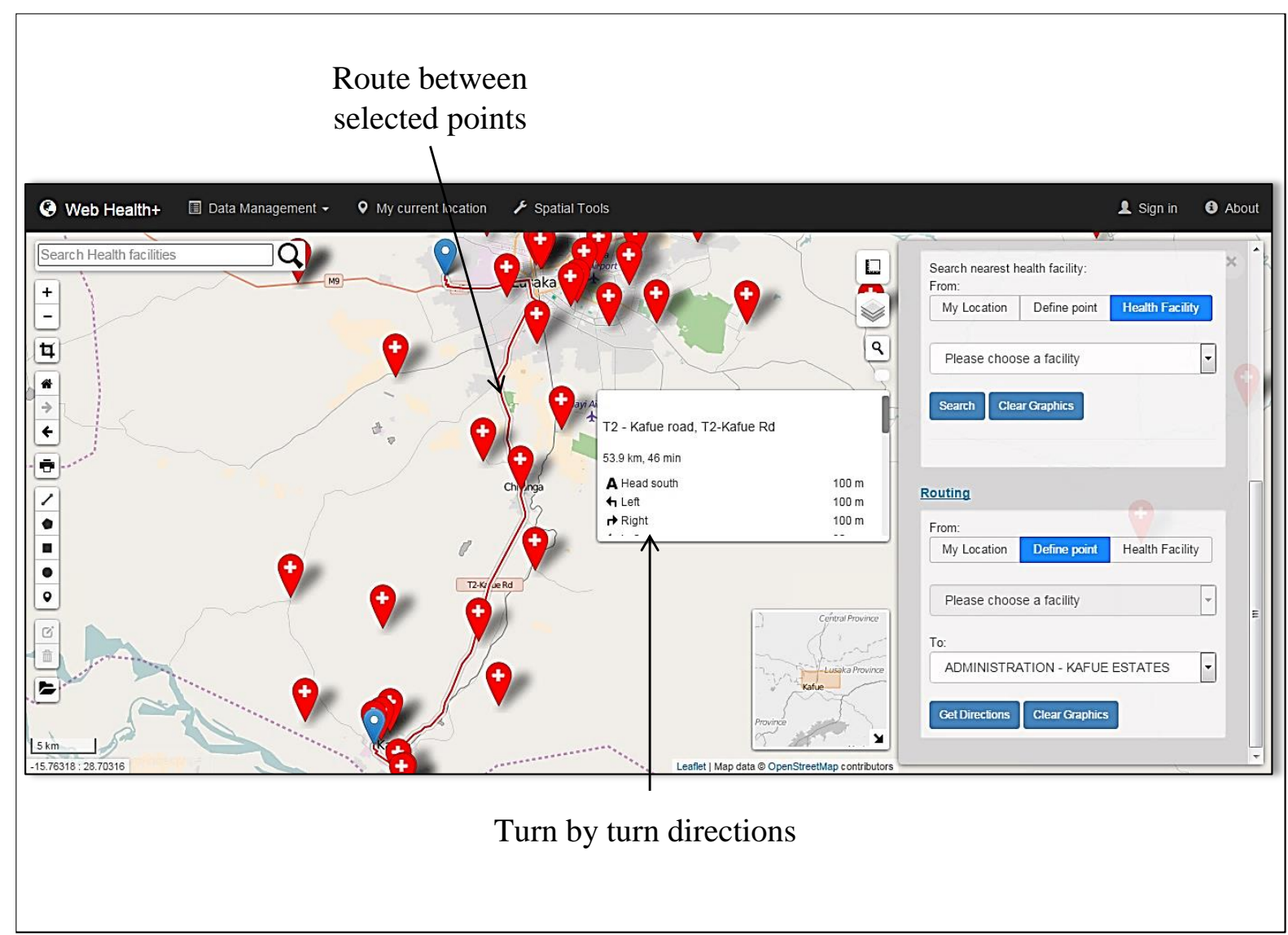

Figure 5. Routing tool

\subsubsection{Point buffering}

This tool enables users to search for health facilities that are within a specified radius from a user's current location, a user defined point or another health facility. The health facilities within the buffer region are highlighted and a count of the facilities within that buffer region is also given. This facility will aid in health facility location planning where planners can determine the number of health facilities within a specified distance of any of the above described points. Figure 6 shows the point buffering function within the health facilities web GIS. 


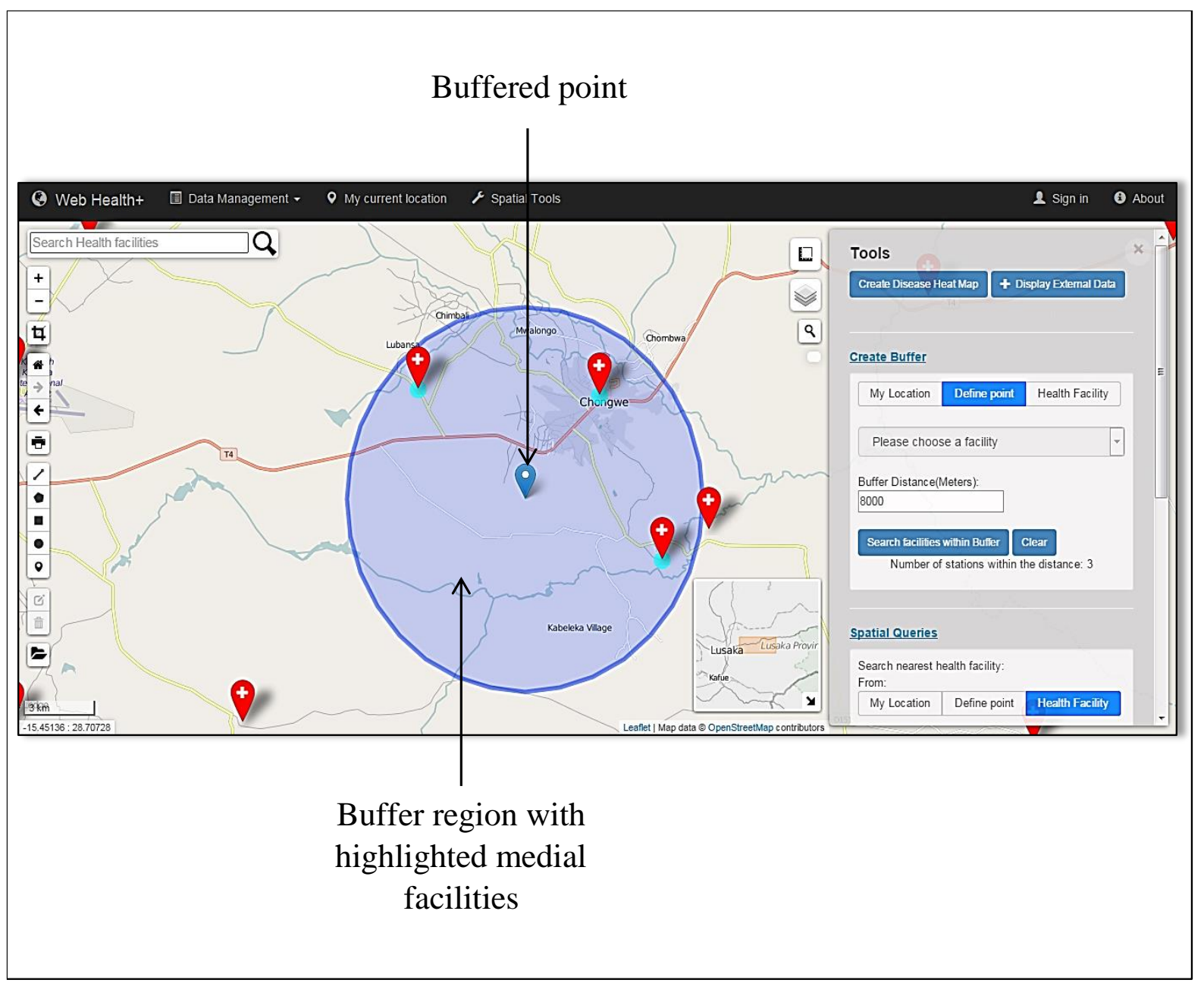

Figure 6. Point buffering tool

\subsection{System Tools}

\subsubsection{Geocode Search}

This tool transforms an address or the name of a place into latitude and longitude. The geocoding tool within the health facilities web GIS leverages the Open Street Map geocoding engine to enable users to search for locations using the name of the place. This tool will aid both health managers and the public to quickly find areas of interest to them within the system.

\subsubsection{Heat map generation}

The heat map generation tool helps to better visualise the concentrations of point data, thus in the health facilities web GIS heat map generation was implemented for disease location sites to aid in monitoring of disease outbreaks. Once a heat map is generated the map may be saved for reporting purposes as an image. Figure 7 shows the heat map generation function. 


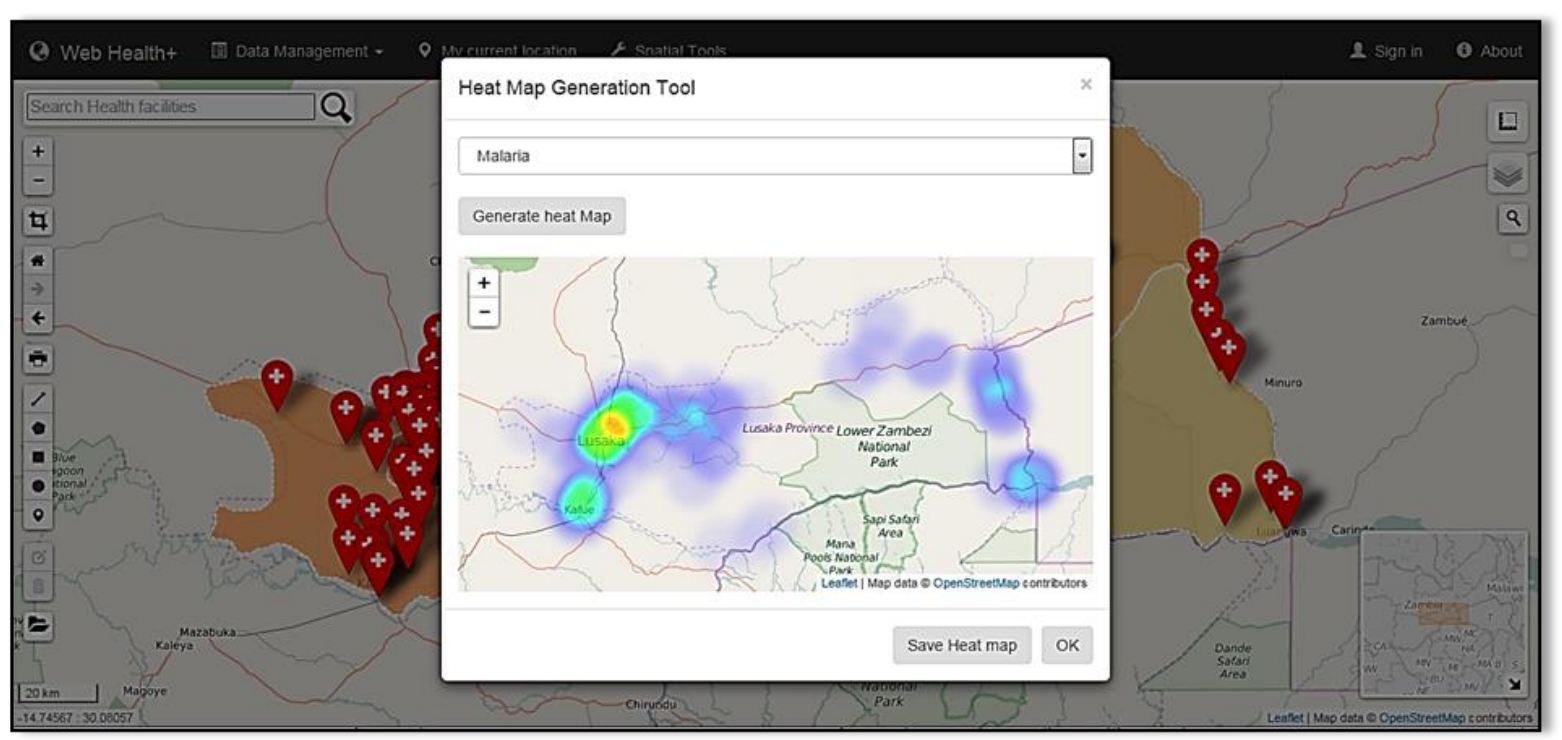

Figure 7. Heat map generation tool

\subsubsection{Health Facility Search}

The web based GIS enables users to search for health facilities by name within the system and then identifying the facility on the map. Once a search is done clicking a result will zoom onto the selected health facility and highlights the facility.

\subsubsection{Drawing and measurement tools}

The system has integrated drawing tools that enable users to draw lines, circles, polygons and points. Editing and deleting functions for the drawn geometries are included. The drawing tools will enable users to highlight certain features for reporting purposes. The web based GIS also has area and distance measurement tools.

\subsubsection{Layer overlay}

The web GIS enables the user to import external layers into the system for visualisation together with the health facilities and diseases layers. The system can integrate several data formats such as GeoJSON and KML (Keyhole Markup Language). The web GIS also has the ability to switch base maps. Users have the ability to choose either street maps (black and white Street or colour) or use a satellite image collage as a base map.

\subsection{Data collection functionality}

The system has functionality to add a new health facility as well as edit and delete a health facility from the database. The system also includes a form to capture the location and attributes of a disease outbreak into the database. The location capture system is similar to that of the new health facility form. There are also forms that enable users to edit and delete a database entry. 


\subsection{System Security}

The system includes a secure user authentication system where only registered users can add, edit and delete data. The health facilities web GIS will be accessed by two categories of users namely health administrators and members of the public. The home screen for both user groups will be the same, with access to data capturing and editing forms being restricted by a user authentication system. The authentication system prevents members of the public from accessing any potentially sensitive information. The security system employs password hashing and a brute force function to prevent malicious attacks on the system.

\section{Conclusions}

The web GIS development process faces new challenges such as technology innovations, voluminous data transfer rates and non-specialist users (Alesheikh et al., 2002). Thus this research has endeavoured to overcome these challenges by innovating on the conventional web GIS architecture and adding client side processing to minimise the volume of data transferred between the browser and the web server. The research has also developed a system with an intuitive and streamlined user interface to enable non specialist users to operate the system without any additional training. The research has managed to meet the set out goals of developing a tool for monitoring and evaluation of health facilities and has also managed to develop a portal for public interaction with health facilities spatial information.

The system developed in this research is web based and will work in any browser and on any device, regardless of screen size, thus vital data can be collected and forwarded using mobile phones leveraging the vast coverage of cellular mobile networks to access the internet. This will enable rural health facilities without computers and fixed line internet connections to access as well as collect data and forward it authorities in a timely manner.

\section{REFERENCES}

Alesheikh, A.A., Helali, H. \& Behroz, H.A. 2002, 'Web GIS: technologies and its applications', Symposium on geospatial theory, processing and applications, vol. 15.

Budge, A., Benedict, K. \& Hudspeth, W. 2006, 'Developing Web-based Mapping Services for Public Health', ISPRS journal, vol. 36, no. 4-B, pp. 565-9.

Church, R.L. 2002, 'Geographical information systems and location science', Computers \& Operations Research, vol. 29, no. 6, pp. 541-62.

Elsabawy, M.N.E.I. 2013, 'Medical Geography in Egypt: As an Interdisciplinary Subfield', Journal of Educational and Social Research, vol. 3, no. 5, p. 109.

Fu, P. \& Sun, J. 2011, Web GIS: Principles and Applications, illustrated edn, ESRI Press 
Glass, G.E., Aron, J.L., Ellis, J.H. \& Yoon, S.S. 1993, 'Applications of GIS technology to disease control'.

Gotz, C., Frates, J. \& Weschler, S. 2009, 'Geographic Information Systems (GIS) in health organizations: emerging trends for professional education', The California Geographer, vol. 49, pp. 87-106.

Green, C. 2012, 'Geographic Information Systems and Public Health: Benefits and Challenges', Purple Paper.

Johnson, C.P. \& Johnson, J. 2001, 'GIS: a tool for monitoring and management of epidemics', Map India.

Mesgari, M. \& Masoomi, Z. 2008, 'GIS applications in public health as a decision making support system and it's limitation in Iran', World appl Sci J (Supple 1), vol. 3, pp. 73-7.

Musa, G.J., Chiang, P.-H., Sylk, T., Bavley, R., Keating, W., Lakew, B., Tsou, H.-C. \& Hoven, C.W. 2013, 'Use of GIS mapping as a public health tool--from cholera to cancer', Health Services Insights, vol. 6, p. 111.

Putz, S. 1994, 'Interactive Information Services Using World-Wide Web Hypertext', paper presented to the First International Conference on World-Wide Web, Geneva, Switzerland.

Tahir, A. 2010, 'Application of GIS IN Health Manangement and Analysis', National Geospatial Information Symposium ed. M. Masniyom, vol. 4, Malaysian Center for Geospatial Data Infrastructure, Putrajaya.

Zeiller, M. 2010, 'Modeling our world: the ESRI guide to Geodatabase concepts', ESRI Press, Redlands. 